PAPER

\title{
The role of dominant striatum in language: a study using intraoperative electrical stimulations
}

\author{
S Gil Robles, P Gatignol, L Capelle, M-C Mitchell, H Duffau
}

J Neurol Neurosurg Psychiatry 2005;76:940-946. doi: 10.1136/jnnp.2004.045948

See end of article for authors' affiliations

Correspondence to: Dr Hugues Duffau, Service de Neurochirurgie, Hôpital de la Salpêtrière, $47-83$ Bd de l'hôpital, 75651

Paris Cedex 13, France; hugues.duffau@ psl.ap-hop-paris.fr

Received 18 May 2004 In revised form

3 September 2004

Accepted 10 October 2004
Background: The role of the striatum in language remains poorly understood. Intraoperative electrical stimulation during surgery for tumours involving the caudate nucleus or putamen in the dominant hemisphere might be illuminating.

Objectives: To study the role of these structures in language, with the aim of avoiding postoperative definitive aphasia.

Methods: 11 patients with cortico-subcortical low grade gliomas were operated on while awake, and striatal functional mapping was done. Intraoperative direct electrical stimulation was used while the patients carried out motor and naming tasks during the resection.

Results: In five cases of glioma involving the dominant putamen, stimulations induced anarthria, while in six cases of glioma involving the dominant caudate, stimulations elicited perseveration. There was no motor effect. The striatum was systematically preserved. Postoperatively, all patients except one had transient dysphasia which resolved within three months.

Conclusions: There appear to be two separate basal ganglia systems in language, one mediated by the putamen which might have a motor role, and one by the caudate which might have a role in cognitive control. These findings could have implications for surgical strategy in lesions involving the dominant striatum.
D espite improved knowledge of the functional role of the striatum in motor function, ${ }^{1-4}$ memory, ${ }^{5}$ attention, ${ }^{6}$ and behaviour, ${ }^{7}$ the exact implication of this structure in language remains poorly understood. Indeed, although many lesional studies have reported language disorders following damage of the basal ganglia in the dominant hemisphere $e^{8-14}$ - in particular speech apraxia and dysarthria after lesion of the lentiform nucleus ${ }^{15-18}$ and perseveration after caudate lesions ${ }^{19}{ }^{20}$-most of these injuries, especially stroke, involve not only the striatum but also the surrounding areas such as the capsulo-thalamic structures. Thus several investigators have attributed aphasia to damage to the communicating fibres ${ }^{1-13} 3^{15-17}$ or to damage to the dominant thalamus, which is well known to be implicated in language. ${ }^{21-24}$

Recent functional neuroimaging studies support a likely role for the dominant striatum in language, as activations were found during various different tasks such as speech, ${ }^{25} 26$ syntactic processing, ${ }^{27}{ }^{28}$ lexical processing, ${ }^{29}$ word memorisation, ${ }^{30}$ word retrieval, ${ }^{31}$ and writing. ${ }^{32}$ However, these methods provide only a statistical evaluation of indirect data, with an imperfect sensitivity and specificity for language mapping $^{33}$ — as recently demonstrated by correlation studies with electrophysiological techniques, which showed agreement between functional magnetic resonance imaging (fMRI) and intraoperative stimulation in only $66 \%$ of cases. ${ }^{34}$

In this study, we used the technique of intraoperative electrical stimulation on awake patients, previously described as representing a precise and reliable method of direct mapping of the language cortical areas ${ }^{35-37}$ and subcortical pathways,,$^{38}$ and we applied these stimulations at the level of the dominant striatum during surgery for low grade gliomas involving the caudate nucleus or putamen. Our goal was to study the potential implication of these structures in language, and thus - on the basis of a better understanding of their pathophysiology - to minimise the risk of permanent postoperative deficit while optimising the quality of resection.

\section{METHODS}

\section{Subjects}

Among a series of 100 patients operated on under local anaesthesia at the Salpêtrière Hospital between 1996 and 2003 for a low grade glioma affecting the language regions, we selected 11 in whom surgical resection involved the dominant striatum.

Preoperatively, all patients had a neurological examination. Language was tested using a French standardised adaptation $^{40}$ of the Boston diagnostic aphasia examination (BDAE). ${ }^{41}$ Hemispheric dominance was defined using a standardised neuropsychological questionnaire. ${ }^{42}$

The topography of the tumour was accurately analysed on preoperative magnetic resonance imaging (MRI; Tl weighted and spoiled gradient images obtained before and after gadolinium enhancement in the three orthogonal planes, T2, and FLAIR weighted axial images).

\section{Intraoperative mapping}

The 11 patients underwent surgery under local anaesthesia so that functional cortical and subcortical mapping could be carried out using direct brain stimulations. This method, including the electrical parameters and the intraoperative clinical tasks, has been described by us previously. ${ }^{39}$ Briefly, a bipolar electrode with $5 \mathrm{~mm}$ spaced tips delivering a biphasic current (pulse frequency $60 \mathrm{~Hz}$; single pulse phase duration $1 \mathrm{~ms}$; amplitude 2 to $8 \mathrm{~mA}$ ) (Ojemann cortical stimulator 1 , Radionics Inc, Burlington, Massachusetts, USA) was applied on the brain of conscious patients. No neuronavigation system was used, because of the risk of brain shift in this type of voluminous tumour. We preferred to use a real time

Abbreviations: BDAE, Boston diagnostic aphasia examination; FLAIR, fluid attenuated inversion recovery 
ultrasonographic system so as not only to delineate the tumour before its removal but also to identify residual tumour along the resection plane.

In a first stage, cortical mapping was carried out before any resection, in order to avoid damage to eloquent areas. Sensorimotor mapping was done first, to confirm a positive response-for example, the induction of movement or paraesthesiae in the contralateral hemibody when the primary sensorimotor areas were stimulated in a patient at rest. The patient was then asked to count (in order from 1 to 10 , and so on) and to name pictures (preceded by "this is a..."), so as to map the cortical language sites known to be inhibited by electrical stimulation using the parameters described above. ${ }^{36}$ For the naming task, we used the DO 80 , which consists of 80 black and white pictures selected according to variables such as frequency, familiarity, age of acquisition, and level of education..$^{43}$ The patient was never informed when the brain was stimulated. The duration of each stimulation was four seconds. At least one picture presentation without stimulation separated each stimulation, and no site was stimulated twice in succession, to avoid seizures. Each cortical site (size $5 \times 5 \mathrm{~mm}$, determined by the spatial resolution of the probe) of the whole cortex exposed by the bone flap was tested three times. It is nowadays accepted, since the seminal publication of Ojemann et al, ${ }^{37}$ that three tasks are sufficient to assess whether a cortical site is essential or non-essential for language, as determined by the generation of speech disturbances during three stimulations, with normalisation of language as soon as the stimulation is stopped. It should be noted that this limitation of the number of trials takes account of the time limitation imposed by the surgical procedure because the patient is awake. The type of language disturbance was defined by a speech therapist who was present in the operative room during the functional mapping. Each eloquent area was marked using a sterile number tag on the brain surface, and its location was correlated with the anatomical landmarks (sulci, gyri, tumour boundaries) previously identified by ultrasonography.

During a second surgical stage, the tumour was removed, with alternating resection and subcortical stimulation. The functional pathways were followed progressively from the cortical eloquent sites already mapped to the full depth of the resection as far as the striatum, which was stimulated in all 11 patients using the same methodology. The patient was asked to continue to carry out both motor tasks (repeated opening and closing of the non-dominant hand) and language tasks (picture naming) when the resection approached the subcortical language structures (white fibres and grey nuclei). These were identified by speech inhibition during stimulation in the same way as at the cortical level. ${ }^{38}$ To achieve the optimum tumour removal consistent with preservation of functional areas, all resections were continued until eloquent structures were encountered around the surgical cavity, and were then terminated along functional boundaries.

The postoperative neurological outcome was assessed systematically immediately after the operation and at three months, using the same language tasks as were used preoperatively. A control MRI examination was carried out in all cases, immediately after surgery and at three months. This imaging allowed us first to evaluate the quality of the glioma removal, and second to analyse where the resection had stopped in relation to the location of the striatum.

\section{RESULTS}

The clinical, radiological, and surgical characteristics of the 11 patients are summarised in table 1 .

\section{Clinical presentation}

The series consisted of eight men and three women, ranging in age from 25 to 52 years. Nine patients were right handed (four with a score of +100 , five with a score of +90 ), and two were left handed $(-80)$. The presenting symptoms were partial seizures with transient language disturbances in four cases, and generalised seizures in seven. Preoperative neurological testing was normal except for a moderate cognitive disorder in patient 3 and slight dysarthria in patient 5. There was no other language disorder involving spontaneous speech, auditory comprehension, word generation, naming, repetition, reading, or writing, according to the BDAE criteria.

\section{Preoperative MRI}

All the tumours appeared as Tl weighted hypointense and T2/FLAIR weighted hyperintense lesion, without enhancement after gadolinium administration.

The 11 tumours were located in the dominant hemisphere-that is, nine on the left side, and two on the right (in the left handed patients). Five lesions were invading the left insular lobe with involvement of the extrema capsule, the claustrum, and the external capsule up to the putamen (patients 1, 2, 3, 4, and 5) (fig 1A). Six tumours were invading the frontomesial precentral structures, with involvement of the subcortical pathways up to the head of the caudate nucleus-four left sided lesions (in patients 6, 8, 9, and 10) (fig 2A), and two right sided (in patients 7 and 11 ).

\section{Operative findings}

The surgical procedure under local anaesthesia was well tolerated by all 11 patients. In all cases, language structures were clearly identified. The results of the striatal mapping differed according to the subregion stimulated.

\section{Insular lesions}

Cortical mapping over the insular cortex showed no response, while stimulation of the frontal operculum elicited speech arrest in all five patients (and also during stimulation of the superior temporal gyrus in two cases). Following insular resection, stimulation of the deep white matter in the posterior part of the cavity induced dysarthria in four cases and anomia in one. More anteriorly, when the resection in the depth of the cavity came into contact with the grey nuclei, which were easily identified because of the change in tissue colour, texture, and vasculature in comparison with the white matter, electrical stimulation of the lateral part of the anterior lentiform nucleus elicited a clear anarthria in all five patients; they were totally unable to articulate, or even to generate the slightest phonation (fig 1B). This anarthria occurred systematically during each stimulation and resolved immediately at the end of stimulation. There was no disruption of motor function during stimulation of the lentiform nucleus, as the patient continued with regular opening and closing of the hand, and there was no facial contraction during the period of inability to articulate. Tumour removal was therefore systematically interrupted at this level.

\section{Fronto-mesial precentral lesions}

Cortical mapping allowed the identification of naming sites within the premotor areas in all six patients. Stimulation of the corresponding subcortical fibres during the resection also elicited anomia laterally (with motor responses posteriorly owing to stimulation of the pyramidal pathways). At the end of the resection, after opening the frontal horn of the ventricle, stimulation of the lateral wall of this hornwhere the supero-medial part of the head of the caudate nucleus was clearly identifiable owing to its colour and 


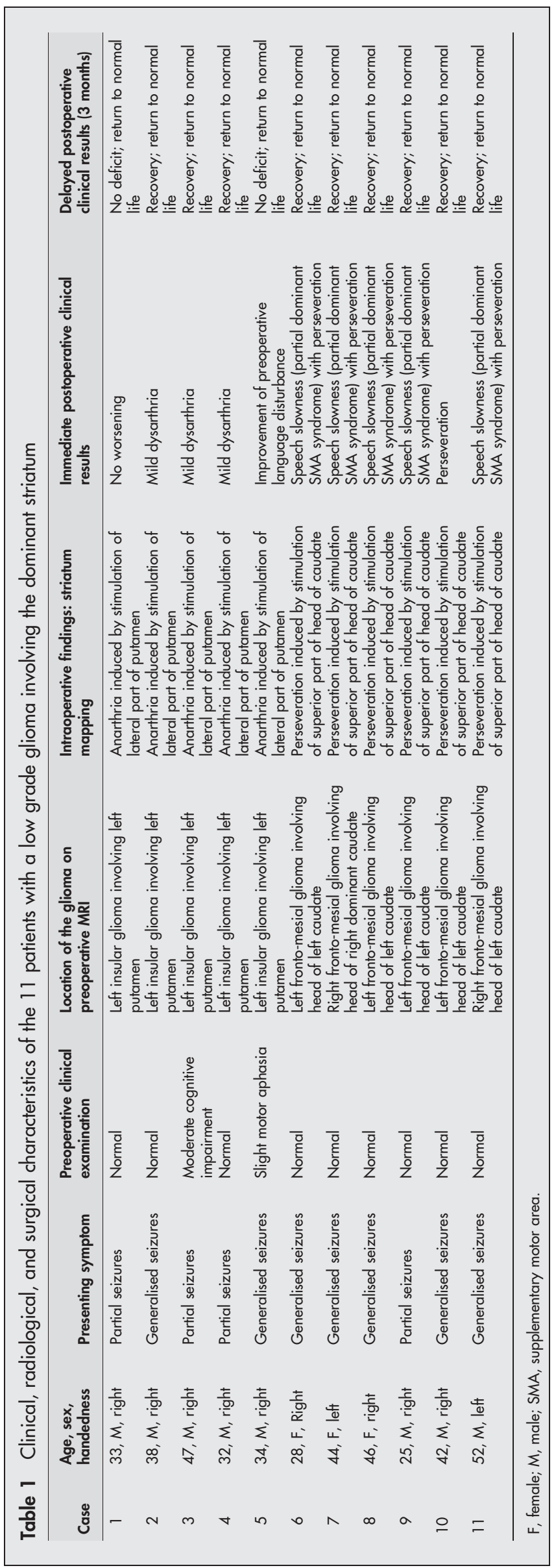

location-induced perseveration in all six patients (four in the left hemisphere (fig $2 \mathrm{~B}$ ), and two in the right). This means that during the naming task the patient repeated the previous and not the current item. Again, perseveration occurred systematically during each stimulation and resolved immediately at the end of stimulation. There was no disruption of motor function during caudate stimulation, as the patient continued with regular opening and closing of the hand, and there was no facial contraction during the period of inability to name. Resection was therefore stopped at this level.

\section{Clinical results}

There was no postoperative sensorimotor deficit. However, nine patients had transient slight to moderate language worsening postoperatively:

- three patients (Nos 2, 3, and 4) operated on for a left insula glioma experienced transitory dysarthria;

- five patients (Nos 6, 7, 8, 9, and 11) operated on for a glioma invading the dominant fronto-mesial structures had slowness of speech corresponding to a transitory "dominant supplementary motor area syndrome," as previously described, ${ }^{44}$ with perseveration;

- one patient (No 10), with a more anterior frontomesial glioma (in front of the supplementary motor area), experienced transient perseveration.

All symptoms disappeared within 10 days to three months.

One patient (No l) with a left insular tumour had no postoperative language worsening, while the patient (No 5) who experienced mild preoperative dysarthria improved immediately after surgery.

\section{Radiological results}

In all cases, postoperative MRI showed that the cavity came into the contact with the striatum. In the five insular lesions, the resection was stopped at the level of the lateral part of the left putamen (fig lC). Owing to tumour infiltration in the subcortical areas in four patients, the resections were total in one case and subtotal in four.

In the six fronto-mesial precentral lesions, lesion removal was interrupted at the level of the head of the caudate nucleus - on the left side in four cases (fig 2C) and on the right side in two. The resections were total in three patients and subtotal in three.

The results of the histopathological examination revealed a low grade glioma (World Health Organisation grade II) in all cases. No patient had chemotherapy or radiotherapy.

\section{DISCUSSION}

In this study we used the technique of intraoperative direct electrical stimulation in conscious patients. This is known to represent a safe, accurate, reliable, and reproducible method of real time identification of the cortical and subcortical (white matter and grey nuclei) structures essential for language function during surgical resection of tumours. ${ }^{39}$ Electrical mapping has been shown to be a valuable adjunct in decreasing postoperative morbidity while improving the quality of resection, especially in brain tumour surgery, producing better outcomes than surgery without mapping. ${ }^{35}{ }^{39}$ This technique also allows analysis of the type of language disturbance induced by each stimulation, and then correlation of the clinical symptoms with the location 

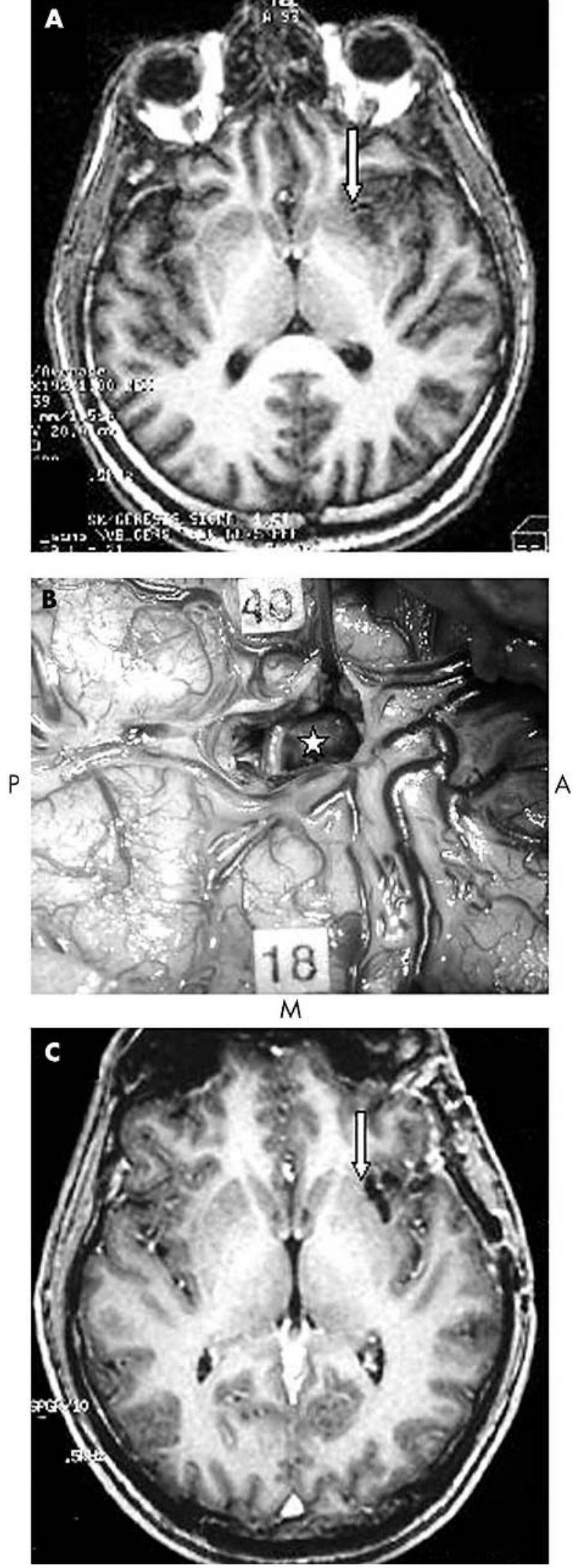

Figure 1 (A) Preoperative axial enhanced $\mathrm{Tl}$ weighted magnetic resonance imaging (MRI), showing a left insular low grade glioma involving the anterolateral part of the putamen (arrow). (B) Intraoperative photograph following tumour removal. This view shows an inverted left hemisphere owing to surgical positioning $(A$, anterior; $P$, posterior; $M$, midline). The eloquent cortical sites which elicited language disturbances when stimulated are marked by the numbers 18 (frontal of the site stimulated. This anatomo-functional study can help determine different eloquent subregions within a wider area, here the dominant striatum. In practice, macroscopic identification of the putamen or the caudate was anatomically very clear in all cases, as these structures are easily distinguishable from the white matter. However, the motive for using subcortical stimulation was to identify specific striatal functions, particularly for language, in order to avoid permanent postoperative deficits. Finally, although all our patients had structural lesions, language disorders only occurred when stimulating at specific electrode positions. In addition, motor responses to cortical stimulation had a distribution (hand/finger superior, face/mouth inferior) appropriate for the primary motor cortex. These findings suggest that our observations accurately reflected the functional anatomy of the regions tested, as we previously reported in other brain areas involved by low grade gliomas. $^{36} 38$

To our knowledge, few data are currently available on striatal mapping, owing to the fact that gliomas involving this structure are rare and generally not operated on except in the non-dominant hemisphere. ${ }^{45}$ Furthermore, although chronic stimulation of the basal ganglia (left or right) is often used in the treatment of movement disorders, the electrode is generally implanted within the subthalamic nucleus, the pallidum, or the thalamus ${ }^{46}$-allowing improved understanding of the pathophysiology of these structures, in particular for language ${ }^{21}$ - but only exceptionally in the striatum. Striatal stimulation has been used for epilepsy treatment, but little attention has been paid to striatal mapping. ${ }^{47}$

Our results are strongly in favour of a key role of the dominant striatum in language, anatomically clearly identified in all 11 patients. Indeed, in all the patients, stimulation of this structure systematically elicited language disturbances which led to termination of tumour removal. Moreover, the functions attributed to the putamen and caudate seem to be language specific, as there were no facial or limb motor effects during stimulation. Control MRI showed that all resections came into contact with the striatum, thus confirming that the language disorders described intraoperatively were definitely caused by stimulation of the putamen and caudate nucleus and not by stimulation of the language fibres. The latter induces different symptoms from those elicited during striatal mapping, as we have shown previously using the same methodology. ${ }^{38}$ Conversely, the fact that 10 of the 11 patients had transient postoperative worsening of language function while intraoperative testing was normal did not allow us to differentiate between dysphasia caused by resection near the cortical language sites (with the occurrence in five cases of a typical supplementary area syndrome), or near the white fibres, or near the striatum (because of transient post-surgical oedema). Interestingly, despite the rarity of this kind of study, Van Buren ${ }^{48}$ observed in 1963, during surgery for basal ganglia disorders, that lower range electrical stimulation at the level of the head of the caudate nucleus induced a "disturbance in which the impulse to speak has been dulled or forgotten".

Another important result of this study is that the dominant striatum rather than the left striatum was shown to be implicated in language, as at the cortical level. Indeed,

site, inducing speech arrest) and 40 (temporal site, inducing anomia). The resection first involved the anterior insula, and then came into contact with the dominant putamen in the depth of the cavity; at this level (star), subcortical stimulation induced anarthria, so the surgical procedure was stopped. (C) Postperative axial enhanced T1 weighted MRI, confirming that the resection was interrupted at the point of contact with the lateral part of the left anterior putamen (arrow). 

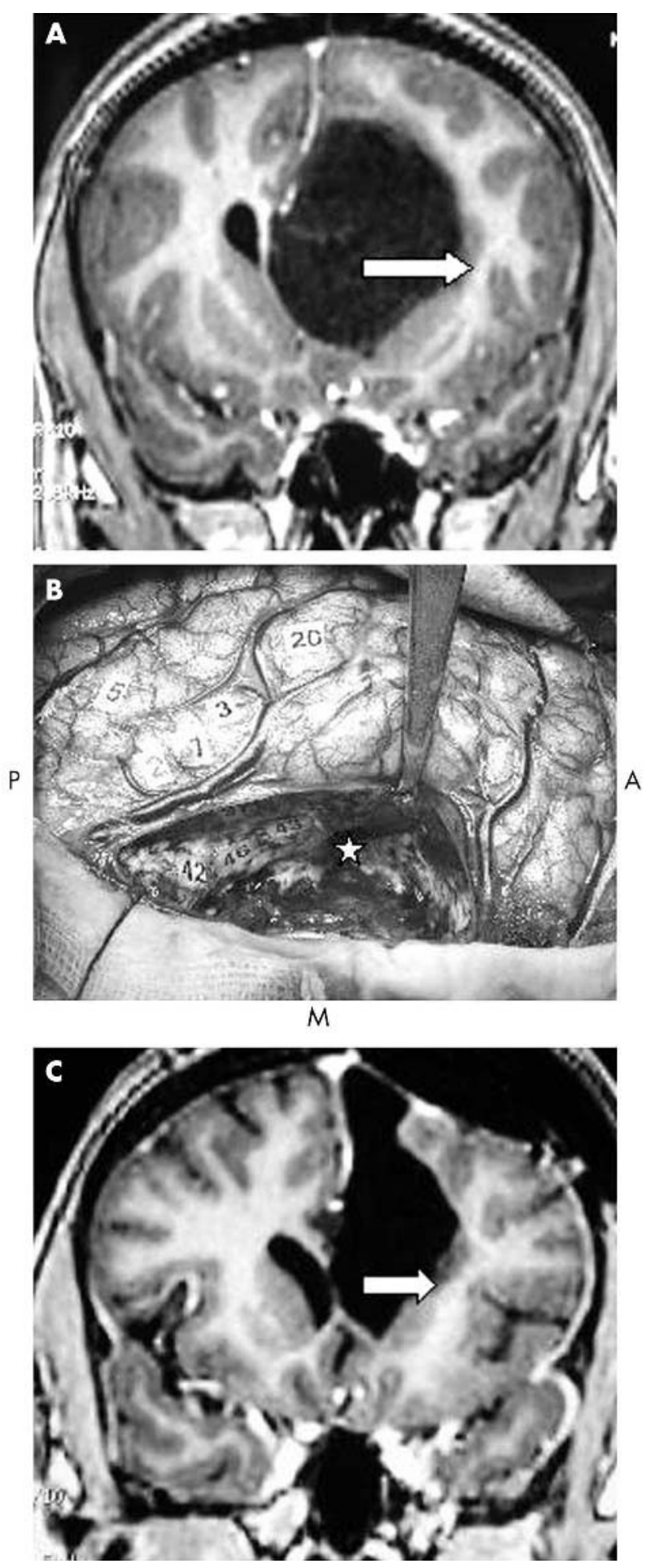

Figure 2 (A) Preoperative coronal enhanced T1 weighted magnetic resonance imaging (MRI), showing a left fronto-mesial low grade glioma involving the superior part of the head of the caudate nucleus (arrow). (B) Intraoperative photograph following tumour removal. This view shows an inverted left hemisphere owing to surgical positioning $(A$ anterior; $P$, posterior; $M$, midline). The eloquent cortical sites, identified using electrical stimulation, are marked by number tags as follows: 2,1 , 3: primary motor area of the superior limb; 5 : primary somatosensory area of the hand; 20: language site. The resection first involved the fronto-mesial structures, with functional boundaries detected using repeated stimulation of the white matter; the boundaries were formed posteriorly by the pyramidal pathways (tags 42 and 46 ) and laterally by language fibres (tag 43), eliciting anomia when stimulated. The resection then came into the contact with the superior part of the dominant caudate in the depth of the cavity; at this level (star), subcortical stimulation although we previously reported that resection of the nondominant striatum did not induce any permanent postoperative deficit, in particular no aphasia, ${ }^{45}$ in the left handed patients in the present series, electrical mapping identified right sided cortical sites within the premotor area that are involved in language (as in the left hemisphere in right handed patients ${ }^{36}$ ). It also showed the implications of the right head of the caudate nucleus in language, as perseveration was elicited when it was stimulated, in the same was as stimulation of the left caudate nucleus in right handed patients. In addition, language pathways were also detected and followed from the cortex to the striatum.

These data thus argue in favour of the existence of right cortico-subcortical loops including the striatum (not only right cortical sites or right cortico-cortical networks), which are implicated in language in left handed patients. Such a result might lead to a better definition of the hemispheric "dominance" for language, which is still a matter of debate, ${ }^{49}$ based on individual data from cortical and subcortical striatal mapping. Nevertheless, as it is currently well known that there is no a direct linear relation between handedness (evaluated here using the Oldfied questionnaire) and language dominance, another potential interpretation is that subcortical striatum bilaterally could be implicated in language function, at least in some left handers.

A third observation in this study is that the dominant striatum seems to be organised in at least two subregions, each playing a different role in language: the anterior putamen and the head of the caudate nucleus. The anterior putamen could be more specifically involved in the coordination of articulation, because dysarthria or anarthria is elicited when it is stimulated, and two patients experienced transient postoperative articulation disorders following insular glioma resection up to the left putamen. These results are in agreement with those provided by lesional and neurofunctional imaging studies. Indeed, many investigators have reported speech planning disorders after infarction or haemorrhage involving the left putamen. ${ }^{15-18} 20$ Furthermore, positron emission tomography (PET) and fMRI data suggested a participation of the left putamen during repetitions of single words ${ }^{26}$ or single syllables, particularly at lower frequencies. ${ }^{25}$ Klein et $a l^{50}$ also found left putaminal activation when speaking a second language, and hypothesised that this region plays a special role for planning complex motor sequences of articulation. Thus it is conceivable that direct stimulation of the dominant putamen induces anarthria, supporting the view that this structure is widely involved in the motor act of speech, and that the processes immediately prior to the execution of articulation are strongly lateralised (though it is well known that the articulatory muscles receive innervation from both cerebral hemispheres). Indeed, we previously reported that resection of the non-dominant striatum did not elicit any postoperative aphasia or anarthria, despite the fact that the patients were not awake in the preliminary study ${ }^{45}$

The supero-medial part of the head of the caudate nucleus seems to be most implicated in the control of language (selection/inhibition), as perseveration is elicited when it is stimulated. Moreover, six patients experienced transient perseveration (associated with a dominant supplementary motor area syndrome in five) following the resection of a glioma involving the fronto-mesial structures up to the caudate nucleus. Interestingly, cortical stimulation never elicited perseveration in these patients. Thus it is likely that

induced perseveration, so the surgical procedure was stopped. (C) Postperative coronal enhanced Tl weighted MRI, confirming that the resection was interrupted at the point of contact with the superior and medial part of the head of the left caudate (arrow). 
this symptom was generated directly by a disturbance of caudate function (as an essential epicentre of the network), and not by spread of electrical stimulation. These results are also in accordance with those provided by lesional and neurofunctional imaging studies. Indeed, the role of lesions of the head of the left caudate nucleus in perseverative errors on a picture naming test has already been suggested. ${ }^{19} 20$ Levitt et $a l^{51}$ also reported significant inverse correlations between caudate nucleus volume and the severity of perseveration in working memory tasks in subjects with schizotypic personality disorder. This is consistent with the general function of the caudate in response selection and control. ${ }^{52}$ Moreover, using PET during the learning of a novel motor task that required inhibition of a previously learned motor sequence, Shadmher and Holcomb ${ }^{53}$ showed that perseveration of a competing motor memory may be linked to reactivation of the neural circuit that participated in acquiring that memory-that is, the left putamen and the bilateral dorsolateral prefrontal cortex. Conversely, in subjects without perseveration, motor learning of a novel task again involved the striatum, but this time in the left caudate, which showed changes in regional cerebral blood flow during the reversal of the learning problem when the previously acquired motor memory was successfully gated. On the basis of this model, we can hypothesise that in our series, stimulation of the head of the dominant caudate might inhibit the "inhibitory" role of this structure, thus resulting in reactivation of the neural circuit that participated to the naming of the previous picture (probably involving the putamen), explaining the occurrence of perseveration. Further studies are required to determine more accurately whether intraoperative stimulation elicits disturbance of a specific language network, or whether it interrupts a more general striato-prefrontal "cognitive" (supramodal) loop useful to language.

\section{Conclusions}

Our results are in agreement with the concept of two separate basal ganglia systems proposed by Middleton and Strick ${ }^{54}$ : one mediated by the putamen (the "sensorimotor" part of the striatum, connected to the sensorimotor cortex), which may have a motor role in language, explaining why direct stimulation of this structure inhibits articulatory sequences and thus elicits anarthria; and the other mediated by the head of the caudate nucleus (the "associative" part of the striatum, connected to the prefrontal cortex), which may have a role in cognitive control, explaining why direct stimulation induces failure to inhibit previously learned responses and thus generates perseveration.

Such improved knowledge of the role of each subregion of the dominant striatum in language may have implications in surgical strategy in lesions involving this structure. Indeed, although we previously reported that resection of tumours invading the right non-dominant striatum was possible without inducing any permanent postoperative deficit, ${ }^{45}$ the present studies support the view that the dominant striatum should be preserved, because it still plays a key role in language even when invaded by a low grade glioma, though it is known to be capable of functional reorganisation. ${ }^{55}$ These observations, showing that functional tissue may persist within low grade gliomas at the level of the grey nuclei, complete the series which previously described the same phenomenon at the cortical level. ${ }^{56}$

The practical lesson for the neurosurgeon is that surgery should be undertaken with the patient awake and with electrical language mapping where tumours near to or within the dominant striatum are to be resected. The goal should be to interrupt lesion removal at the point of contact with the cortico-subcortical language loops-that is, at the functional boundaries represented by the cortical language sites-then at the corresponding language pathways, and then at the dominant putamen or caudate nucleus in the depth of the cavity. Complementary cognitive tasks may be considered in the future to further improve the quality of intraoperative striatal mapping.

The present data do not allow us to determine with certainty what would have happened if the language sites detected by stimulation within the dominant striatum had been resected to improve the quality of glioma removal. Indeed, we previously reported that brain areas considered as essential for language could sometimes be resected-in one ${ }^{57}$ or two surgical procedures ${ }^{56}$-because of the functional reshaping mechanisms induced by the lesion or by the surgery itself. ${ }^{58}$ However, the frequent permanent aphasias reported after lesions of the dominant striatum demand caution before making any decision to resect this structureeven though its function could eventually be compensated by other brain areas, as already shown for the right striatum. ${ }^{45}$ Consequently, a multimodal study of this complex area is needed (combining the data obtained using intraoperative electrical mapping and those obtained by preoperative and postoperative functional neuroimaging), to improve our understanding of its integration in language networks, in particular its relation to the other structures involved in speech production, and to apply this knowledge to the surgery of striatal lesions. This work is currently in progress in our institution.

\section{Authors' affiliations}

S Gil Robles, Department of Neurosurgery, Hospital Clinico San Carlos, Universidad Complutense De Madrid, Madrid, Spain

P Gatignol, Departments of Neurology, Hôpital de la Salpêtrière, Paris Cedex 13, France

L Capelle, H Duffau, Departments of Neurosurgery, Hôpital de la Salpêtrière

M-C Mitchell, Departments of Neuroanaesthesiology, Hôpital de la Salpêtrière

Competing interests: none declared

\section{REFERENCES}

1 Alexander GE, DeLong MR, Strick PL. Parallel organization of functionally segregated circuits linking basal ganglia and cortex. Annu Rev Neurosci 1986;9:357-81.

2 Bathia KP, Marsden CD. The behavioral and motor consequences of focal lesions of the basal ganglia in man. Brain 1994;117:859-76.

3 Middleton FA, Strick PL. Basal ganglia and cerebellar loops: motor and cognitive circuits. Brain Res Rev 2000;31:236-50.

4 Parent A, Hazrati L. Functional anatomy of the basal ganglia. I. The corticobasal ganglia-thalamo-cortical loop. Brain Res $\operatorname{Rev} 1995 ; 20: 91-127$.

5 Hay JF, Moscovitch M, Levine B. Dissociating habit and recollection: evidence from Parkinson's disease, amnesia and focal lesion patients. Neuropsychologia 2002;40:1324-34.

6 Habekost T, Bundesen C. Patient assessment based on a theory of visual attention (TVA): subtle deficits after a right frontal-subcortical lesion. Neuropsychologia 2003;41:1171-88.

7 Saint-Cyr JA, Taylor AE, Nicholson K. Behavior and the basal ganglia. Adv Neurol 1995;65:1-28

8 Chenery HJ, Copland DA, Murdoch BE. Complex language functions and subcortical mechanisms: evidence from Huntington's disease and patients with non-thalamic subcortical lesions. Int J Lang Commun Disord 2002;37:459-74.

9 Ho AK, Sahakian BJ, Robbins TW, et al. Verbal fluency in Huntington's disease: a longitudinal analysis of phonemic and semantic clustering and switching. Neuropsychologia 2002;40:1277-84.

10 Kumral E, Evyapan D, Balkir K. Acute caudate vascular lesions. Stroke 1999;30:1734-5.

11 Mega MS, Alexander MP. Subcortical aphasia: the core profile of capsulostriatal infarction. Neurology 1994;44:1824-9.

12 Milhaud D, Magnie MN, Roger PM, et al. Infarction of the caudate nucleus or anterior striato-capsular infarction? Rev Neurol (Paris) 1994;150:286-91.

13 Nadeau SE, Crosson B. Subcortical aphasia. Brain Lang 1997;58:355-402.

14 Pickett ER, Kuniholm E, Protopapas A, et al. Selective speech motor, syntax and cognitive deficits associated with bilateral damage to the putamen and the head of the caudate nucleus: a case study. Neuropsychologia 1998;36:173-88

15 Alexander MP, Naeser MA, Palumbo CL. Correlation of subcortical CT lesion sites and aphasia profiles. Brain 1987;110:961-91. 
16 D'Esposito M, Alexander MP. Subcortical aphasia. distinct profiles following left putaminal hemorrhage. Neurology 1995;45:38-41.

17 Kawanishi M, Kajikawa H, Yamamura K, et al. Aphasia following left putaminal hemorrhage. Statistical analysis of factors affecting prognosis. Neurol Res 2002;24:817-21.

18 Warren JD, Smith HB, Denson LA, et al. Expressive language disorder after infarction of left lentiform nucleus. J Clin Neurosci 2000;7:456-8.

19 Caplan LR, Schmahmann JD, Kase CS, et al. Caudate infarcts. Arch Neurol 1990:47:133-43.

20 Kreisler A, Godefroy O, Delmaire C, et al. The anatomy of aphasia revisited. Neurology 2000;54:1117-23.

21 Johnson MD, Ojemann GA. The role of the human thalamus in language and memory: evidence from electrophysiological studies. Brain Cogn 2000;42:218-30.

22 Kennedy M, Murdoch BE. Chronic aphasia subsequent to striato-capsular and thalamic lesions in the left hemisphere. Brain Lang 1993;44:284-95.

23 Kumar R, Masih AK, Pardo J. Global aphasia due to thalamic hemorrhage: a case report and review of the literature. Arch Phys Med Rehabil 1996;77:1312-15.

24 Troster Al, Wilkinson SB, Fields JA, et al. Chronic electrical stimulation of the left ventrointermediate (Vim) thalamic nucleus for the treatment of pharmacotherapy-resistant Parkinson's disease: a differential impact on access to semantic and episodic memory? Brain Cogn 1998;38:125-49.

25 Wildgruber D, Ackermann H, Grodd W. Differential contributions of motor cortex, basal ganglia, and cerebellum to speech motor control: effects of syllable repetition evaluated by fMRI. Neurolmage 2001;13:101-9.

26 Wise RSJ, Greene J, Buchel C, et al. Brain regions involved in articulation. Lancet 1999;353:1057-61.

27 Moro A, Tettamanti M, Perani D, et al. Syntax and the brain: disentangling grammar by selective anomalies. Neuroimage 2001;13:110-18.

28 Ullman MT. A neurocognitive perspective on language: the declarative/ procedural model. Nat Rev Neurosci $2001 ; 2: 717-26$.

29 Kotz SA, Cappa SF, von Cramon DY, et al. Modulation of the lexical-semantic network by auditory semantic priming: an event-related functional MRI study. Neuroimage 2002;17:1761-72.

30 Demonet JF, Celsis P, Agniel A, et al. Activation of regional cerebral blood flow by a memorization task in early Parkinson's disease patients and norma subjects. J Cereb Blood Flow Metab 1994;14:431-8.

31 Rosen HJ, Ojemann JG, Ollinger JM, et al. Comparison of brain activation during word retrieval done silently and aloud using fMRI. Brain Cogn 2000;42:201-17.

32 Siebner HR, Limmer C, Peinemann A, et al. Brain correlates of fast and slow handwriting in humans: a PET-performance correlation analysis. Eur J Neurosci $2001 ; 14: 726-36$

33 Rutten GJ, Ramsey NF, van Rijen PC, et al. Development of a functional magnetic resonance imaging protocol for intraoperative localization of critical temporoparietal language areas. Ann Neurol 2002;51:350-60.

34 Roux FE, Boulanouar K, Lotterie JA, et al. Language functional magnetic resonance imaging in preoperative assessment of language areas: correlation with direct cortical stimulation. Neurosurgery 2003;52:1335-45.

35 Berger MS. Functional mapping-guided resection of low-grade gliomas. Clin Neurosurg 1995;42:437-52

36 Duffau H, Capelle L, Denvil D, et al. The role of dominant premotor cortex in language: a study using intraoperative functional mapping in awake patients. Neurolmage 2003;20:1425-37.
37 Ojemann GA, Ojemann JG, Lettich E, et al. Cortical language localization in left, dominant hemisphere. An electrical stimulation mapping investigation in 117 patients. J Neurosurg 1989;71:316-26.

38 Duffau H, Capelle L, Sichez N, et al. Intraoperative mapping of the subcortical language pathways using direct stimulations. An anatomo-functional study. Brain 2002;125:199-214.

39 Duffau H, Capelle L, Denvil D, et al. Usefulness of intraoperative electrical subcortical mapping during surgery for low-grade gliomas located within eloquent brain regions: functional results in a consecutive series of 103 patients. J Neurosurg 2003;98:764-78.

40 Mazaux JM, Orgogozo JM. Echelle d'evaluation de l'aphasie adaptee du Boston diagnostic aphasia examination. Paris: EAP Editions Psychotechniques, 1982.

41 Goodglass H, Kaplan E. The assessment of aphasia and related disorders. Philadelphia: Lea and Febiger, 1972.

42 Oldfield RC. The assessment and analysis of handedness: the Edinburgh inventory. Neuropsychologia 1971;9:97-113.

43 Metz-Lutz MN, Kremin H, Deloche G, et al. Standardisation d'un test de denomination orale: controle des effets de l'age, du sexe et du niveau de scolarite chez les sujets adultes normaux. Rev Neuropsychol 1991;1:73-95.

44 Krainik A, Lehericy S, Duffau $\mathrm{H}$, et al. Postoperative speech disorder after medial frontal surgery: role of the supplementary motor area. Neurology 2003;60:587-94

45 Duffau H, Denvil D, Capelle L. Absence of movement disorders after surgical resection of glioma invading the right striatum. J Neurosurg 2002;97:363-9.

46 Benabid AL. Deep brain stimulation for Parkinson's disease. Curr Opin Neurobiol 2003; 13:696-706.

47 Chkhenkeli SA, Chkhenkeli IS. Effects of therapeutic stimulation of nucleus caudatus on epileptic electrical activity of brain in patients with intractable epilepsy. Stereotact Funct Neurosurg 1997;69:221-4.

48 Van Buren JM. Confusion and disturbance of speech from stimulation in vicinity of the head of the caudate nucleus. J Neurosurg 1963;20:148-57.

49 Szaflarski J, Binder J, Possing E, et al. Language lateralization in left-handed and ambidextrous people. FMRI data. Neurology 2002;59:238-44.

50 Klein D, Zatorre RJ, Milner B, et al. Left putaminal activation when speaking a second language: evidence from PET. Neuroreport 1994;5:2295-7.

51 Levitt JJ, McCarley RW, Dickey CC, et al. MRI study of caudate nucleus volume and its cognitive correlates in neuroleptic-naive patients with shizotypal personality disorder. Am J Psychiatry 2002;159:1190-7.

52 Brown P, Marsden CD. What do the basal ganglia do? Lancet 1998;351:1801-4

53 Shadmehr R, Holcomb HH. Inhibitory control of competing motor memories. Exp Brain Res 1999;126:235-51.

54 Middleton FA, Strick L. Basal ganglia output and cognition: evidence from anatomical, behavioral, and clinical studies. Brain Cogn 2002;42:183-200.

55 Duffau H, Capelle L, Denvil D, et al. Functional recovery after surgical resection of low grade gliomas in eloquent brain: hypothesis of brain compensation. J Neurol Neurosurg Psychiatry 2003;74:901-7.

56 Duffau H, Denvil D, Capelle L. Long term reshaping of language, sensory, and motor maps after glioma resection: a new parameter to integrate in the surgical strategy. J Neurol Neurosurg Psychiatry 2002;72:511-16.

57 Duffau $\mathrm{H}$, Bauchet L, Lehericy S, et al. Functional compensation of the left insula dominant for language. Neuroreport 2001;12:2159-63.

58 Duffau $\mathbf{H}$. Acute functional reorganisation of the human motor cortex during resection of central lesions: a study using intraoperative brain mapping J Neurol Neurosurg Psychiatry 2001;70:506-13. 